\title{
Revised Standardized Equation for Hydrogen Gas Densities for Fuel Consumption Applications
}

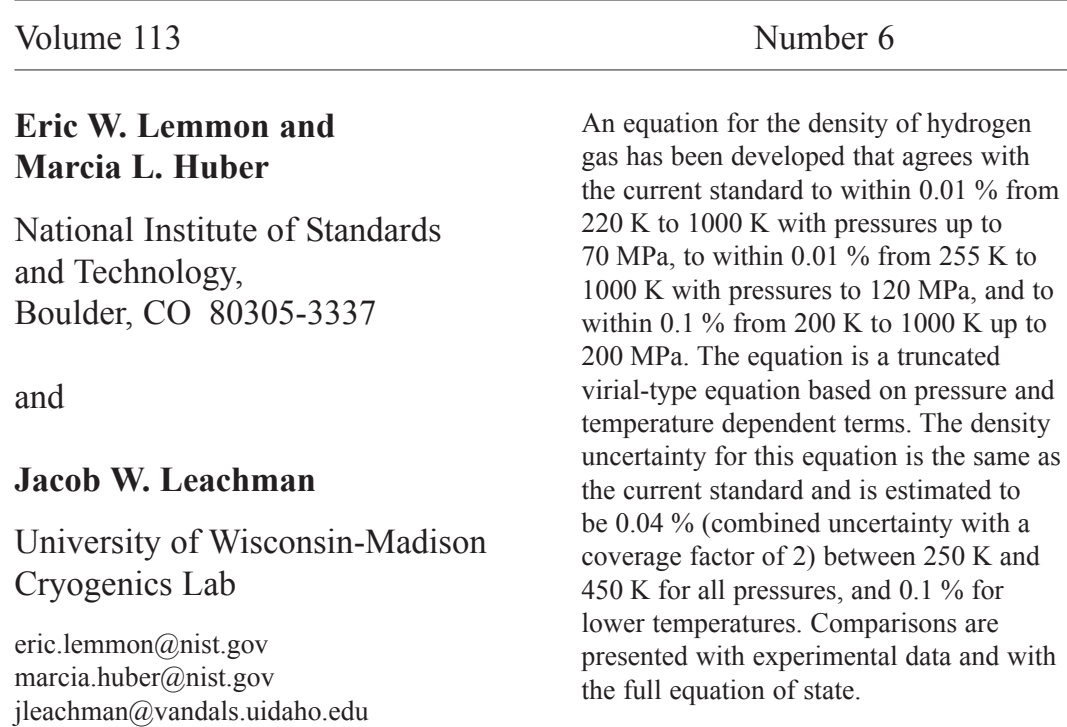

\begin{abstract}
An equation for the density of hydrogen gas has been developed that agrees with $220 \mathrm{~K}$ to $1000 \mathrm{~K}$ with pressures up to $70 \mathrm{MPa}$, to within $0.01 \%$ from $255 \mathrm{~K}$ to $1000 \mathrm{~K}$ with pressures to $120 \mathrm{MPa}$, and to $0.1 \%$ from $200 \mathrm{~K}$ to $1000 \mathrm{~K}$ up virial-type equation based on pressure and temperature dependent terms. The density uncertainty for this equation is the same as the current standard and is estimated to coverage factor of 2) between $250 \mathrm{~K}$ and $450 \mathrm{~K}$ for all pressures, and $0.1 \%$ for the full equation of state.
\end{abstract}

Key words: density, equation of state; fuel consumption; hydrogen; pressure; temperature.

Accepted: July 22, 2008

Available online: http://www.nist.gov/jres

\section{Introduction}

The equilibrium temperature and pressure of a gas before and after usage within a storage tank of known, and essentially fixed, volume can be used to calculate consumption. Equations of state for calculating the thermodynamic properties generally provide the pressure as a function of density and temperature. In some fuel consumption applications, this form is inconvenient to use since the equation must be solved in an iterative manner in order to provide the density in terms of pressure and temperature. In order to easily calculate gaseous hydrogen fuel consumption in vehicle applications in which temperature and pressure are measured, an equation with these independent properties that agrees with the current standard is desirable.

The former standard for hydrogen was the equation of state by Younglove [1] published in 1982 and was a 32-term expression for pressure as a function of temperature and density, $p(T, \rho)$. This equation for parahydrogen, adapted for use for normal hydrogen, was once considered adequate for density calculations in the region of interest. It is a rather dated standard, and the basic source of the parahydrogen equation is a 1975 National Bureau of Standards (NBS) technical report [2,3]. (NBS is the former name of the National Institute of Standards and Technology, NIST.) The density uncertainty for this equation was estimated as $0.2 \%$ (combined uncertainty with a coverage factor of 2). Some of the older NBS material and a bibliography of hydrogen property information can be found through the NIST hydrogen web site $\mathrm{http} / / /$ www.nist.gov/public_affairs/hydrogen.htm that is part of the larger hydrogen site for the United States Government, http://www.hydrogen.gov.

There have been several recent advances in the state of the art for the thermodynamic properties of hydrogen. New equations of state have been developed in the 
Master's Thesis of Jacob Leachman [4] for normal hydrogen, parahydrogen, and orthohydrogen; these equations will be fully documented elsewhere [5]. These equations replace those presented by Younglove in 1982 and decrease the uncertainty in density in the normal hydrogen equation of state from $0.2 \%$ to $0.04 \%$ in the range from $250 \mathrm{~K}$ to $450 \mathrm{~K}$ with pressures up to $300 \mathrm{MPa}$.

In 2006, Lemmon et al. [6] published a short equation for the density of hydrogen between $220 \mathrm{~K}$ and $400 \mathrm{~K}$ with pressures to $45 \mathrm{MPa}$. This equation calculated density as a function of temperature and pressure and agreed with the Younglove equation of state to less than $0.01 \%$ in density. It is used in SAE Standard J2572, Recommended Practice for Measuring Fuel Consumption and Range of Fuel Cell and Hybrid Fuel Cell Vehicles Fuelled by Compressed Gaseous Hydrogen [6]. With the new equation of state of Leachman, the 2006 work is now out of date and a replacement is needed. The work presented here updates and expands the 2006 work and provides an equation for the density of hydrogen as a function of temperature and pressure, expanding the temperature range to the upper limit used by Leachman and the pressure range to $200 \mathrm{MPa}$. The 2006 work gave additional information on the use of the equation in hydrogen gas consumption applications that is not repeated here.

\section{Hydrogen Equation as a Function of Pressure and Temperature}

The Leachman equation of state from Ref. [4] is a 14-term Helmholtz energy equation written as

$$
\begin{aligned}
\alpha^{\mathrm{r}}(\delta, \tau) & =\sum N_{k} \delta^{d_{k}} \tau^{t_{k}}+\sum N_{k} \delta^{d_{k}} \tau^{t_{k}} \exp \left(-\delta^{l_{k}}\right) \\
& +\sum N_{k} \delta^{d_{k}} \tau^{t_{k}} \exp \left(\eta\left(\delta-\varepsilon_{k}\right)^{2}+\beta\left(\tau-\gamma_{k}\right)^{2}\right)
\end{aligned}
$$

where $\alpha^{\mathrm{r}}$ is the residual Helmholtz energy, $\delta$ is the reduced density $\rho / \rho_{\mathrm{c}}, \tau$ is the reduced inverse temperature $T_{\mathrm{c}} / T$, and the subscript $\mathrm{c}$ denotes the value at the critical point. The other parameters are constants that were determined during the fitting of available property data. Values of the constants can be found in Refs. $[4,5]$. This expression, together with an equation for the ideal gas heat capacity, enables a thermodynamically consistent calculation of many properties of hydrogen in the liquid, vapor, and supercritical phases (density, isochoric and isobaric heat capacities, sound speed, phase boundaries, enthalpy, etc.) from about $14 \mathrm{~K}$ to
$1000 \mathrm{~K}$ with pressures to $2000 \mathrm{MPa}$. This equation is implemented in the REFPROP software [7] and is available through the NIST Chemistry Webbook (http://webbook.nist.gov). Inversion of the equation through iterative solutions is straightforward; nonetheless, direct use of Eq. (1) for hydrogen consumption calculations may not be convenient.

A common equation for the density of gases is based on the virial series, which has a statistical-mechanical basis in terms of the relation between the number of particles interacting and the significance of such multiparticle interactions at a particular gas density. Often, the virial equation is written in the form of an expression for the pressure as a sum of the powers of density multiplied by temperature-dependent virial coefficients. Alternatively, the temperature-dependent virial equation may be expressed in terms of the powers of pressure. For the compressibility factor, $Z=p /(\rho R T)$, this becomes

$$
Z(p, T)=1+\sum_{i=2}^{n} B_{i}^{*}(T) p^{i-1},
$$

where the $B_{i}^{*}(T)$ quantities are the temperaturedependent pressure virial coefficients.

The lower virial coefficients (e.g., the second virial coefficient, $B_{2}^{*}$ ) can be calculated theoretically if the interaction potential between hydrogen molecules is known (e.g., through quantum mechanical calculations). However, the current effort has focused on establishing an equation of the form given in Eq. (2) that agrees with the standard of Ref. [4] to within $0.01 \%$ in density over the range of interest. No attempts were made to determine the virial coefficients theoretically, although experimental values of the second virial coefficient were used in the development of the formulation in Ref. [4].

The specific terms and coefficients were determined by calculating a set of compressibility factor values distributed in $(p, T)$ space with the REFPROP [7] implementation of Eq. (1). These values were then used in a structural optimization/regression algorithm [8] with the system constrained to consider the lowest virial coefficients of Eq. (2) and simple temperature dependences for $B_{i}^{*}(T)$. This structure/parameter space was searched systematically until an equation meeting the criterion of $0.01 \%$ agreement in density was obtained. Virial coefficients up to the sixth power were considered in order to match the isothermal curvature of the hydrogen equation of state above $150 \mathrm{~K}$. Initial fitting considered only integer values of the exponents on pressure. However, it was only possible to achieve high 
accuracy and expand the range of coverage by using noninteger values. The use of noninteger exponents has a negative impact on only the third and higher derivatives of the equation, which is not of concern in this work. The resulting coefficients and exponents were truncated to the extent allowed to retain agreement with the equation of state within the specified tolerance. The equation was examined to ensure reasonable extrapolation over a broader temperature and pressure range; however, derivatives were not examined as was done with the original equation of state. The equation given here is intended for use only in density calculations over the range specified. The underlying equation of state [4] should be used for all other calculations.

The resulting expression for the compressibility factor is

$$
Z(p, T)=\frac{p}{\rho R T}=1+\sum_{i=1}^{9} a_{i}\left(\frac{100 \mathrm{~K}}{T}\right)^{b_{i}}\left(\frac{p}{1 \mathrm{MPa}}\right)^{c_{i}}
$$

The constants associated with Eq. (3) are given in Table 1. The equation and its constants are given for pressure in megapascals $(\mathrm{MPa})$ and temperature in kelvins $(\mathrm{K})$. The mass of diatomic hydrogen and the molar gas constant given in Table 1 are from the most recent tabulations of such information $[9,10]$. The molar mass is given to aid in conversions from molar to mass density. Test points that can be used to verify algorithms based on Eq. (3) are given in Table 2. More digits are given than the uncertainty in the equation to aid in validation.

Table 1. Constants associated with the density equation for normal hydrogen

\begin{tabular}{lcll}
\hline \hline$i$ & \multicolumn{1}{c}{$a_{i}$} & \multicolumn{1}{c}{$b_{i}$} & $c_{i}$ \\
\hline 1 & 0.05888460 & 1.325 & 1.0 \\
2 & -0.06136111 & 1.87 & 1.0 \\
3 & -0.002650473 & 2.5 & 2.0 \\
4 & 0.002731125 & 2.8 & 2.0 \\
5 & 0.001802374 & 2.938 & 2.42 \\
6 & -0.001150707 & 3.14 & 2.63 \\
7 & $0.9588528 \times 10^{-4}$ & 3.37 & 3.0 \\
8 & $-0.1109040 \times 10^{-6}$ & 3.75 & 4.0 \\
9 & $0.1264403 \times 10^{-9}$ & 4.0 & 5.0 \\
\hline
\end{tabular}

Molar Mass: $M=2.01588 \mathrm{~g} / \mathrm{mol}$

Universal Gas Constant: $R=8.314472 \mathrm{~J} /(\mathrm{mol} \cdot \mathrm{K})$
Table 2. Test points for validating computer code based on Eq. (3)

\begin{tabular}{cccc}
\hline \hline$T(\mathrm{~K})$ & $p(\mathrm{MPa})$ & $Z$ & $\rho(\mathrm{mol} / 1)$ \\
\hline 200 & 1 & 1.00675450 & 0.59732645 \\
300 & 10 & 1.05985282 & 3.78267048 \\
400 & 50 & 1.24304763 & 12.09449023 \\
500 & 200 & 1.74461629 & 27.57562673 \\
200 & 200 & 2.85953449 & 42.06006952 \\
\hline
\end{tabular}

\section{Evaluation of the Equation}

As discussed above, Eq. (3) and the related constants were established by calculating values from the equation of state [4] and regressing the structure and coefficients using these values. Equation (3) was in turn evaluated through a more thorough comparison with the equation of state and by comparing with available experimental data for the density of hydrogen over the range of the equation.

Densities were computed with the new formulation (Eq. (3) with constants from Table 1) and compared with densities obtained from the equation of state of Leachman [4] for several temperature and pressure ranges to assess how well Eq. (3) agrees with the equation of state. Of specific interest is the $(T, p)$ region where densities calculated from Eq. (3) and densities from the full equation of state of Leachman are in agreement to within $0.01 \%$. One million points in the $(T, p)$ region from $220 \mathrm{~K}$ to $1000 \mathrm{~K}$ with pressures to $70 \mathrm{MPa}$, and a second $(T, p)$ region from $255 \mathrm{~K}$ to $1000 \mathrm{~K}$ with pressures to $120 \mathrm{MPa}$, were randomly generated and the results from Eq. (3) were compared with the densities computed from the Leachman equation of state with the REFPROP software [7]. For both of these $(T, p)$ regions the randomly selected points never exceeded $0.01 \%$ deviation in density from the equation of state of Leachman [4]. Over the range of temperatures from $220 \mathrm{~K}$ to $1000 \mathrm{~K}$ with pressures to $70 \mathrm{MPa}$, the average absolute percentage deviation for these million points was $0.0020 \%$, with a standard deviation of $0.0022 \%$. The maximum positive deviation was $0.0075 \%$, and the largest negative deviation was $-0.0046 \%$. The largest deviations occurred at the low temperature limit of $220 \mathrm{~K}$. If the temperature range is limited to $255 \mathrm{~K}$ to $1000 \mathrm{~K}$, Eq. (3) is in agreement to within $0.01 \%$ with the equation of state up to $120 \mathrm{MPa}$. Over this $(T, p)$ range, the average percentage deviation for these million points was $0.0025 \%$, with a standard deviation of $0.0024 \%$. The maximum positive deviation was $0.0097 \%$, and the largest negative deviation was $-0.0026 \%$. 
Figures 1 and 2 are histograms of the results. Figure 1 covers the region $220 \mathrm{~K}$ to $1000 \mathrm{~K}$ at pressures up to $70 \mathrm{MPa}$, while Fig. 2 displays the range $255 \mathrm{~K}$ to $1000 \mathrm{~K}$ at pressures to $120 \mathrm{MPa}$. Figure 1 shows that the largest number of points had a percentage deviation between $0.001 \%$ and $0.0025 \%$; the second largest group had deviations between $0.0025 \%$ and $0.005 \%$. There were no points with deviations larger than $0.0075 \%$. Figure 2 shows that the largest number of points had a percentage deviation between $0.001 \%$ and $0.0025 \%$; the second largest group had deviations between $0.0025 \%$ and $0.005 \%$. There were no points with deviations larger than $0.01 \%$.

The new formulation (Eq.(3) with constants from Table 1) can be extrapolated outside the two ranges discussed above. In the extended region with pressures to $200 \mathrm{MPa}$ but with temperatures higher than $300 \mathrm{~K}$, the maximum deviation (as compared to the equation of state of Leachman [4]) is less than $0.006 \%$. If the lower temperature limit is lowered to $200 \mathrm{~K}$ but with pressures still up to $200 \mathrm{MPa}$, the maximum deviation is $0.1 \%$, and if the lower temperature limit is lowered to $150 \mathrm{~K}$, the maximum deviation is $0.15 \%$. Figure 3 shows this evaluation in more detail.

The second type of evaluation was direct comparison with available experimental data. Figures 4 to 6 show the deviations between densities calculated with Eq. (3) and experimental data in the literature [11-19]. The subplots in these figures include data from various sources grouped in isotherms. A line comparing the representation of Eq. (3) with the equation of state of Leachman [4] is not provided, as it is extremely close to the zero line (within $0.01 \%$, as indicated above).

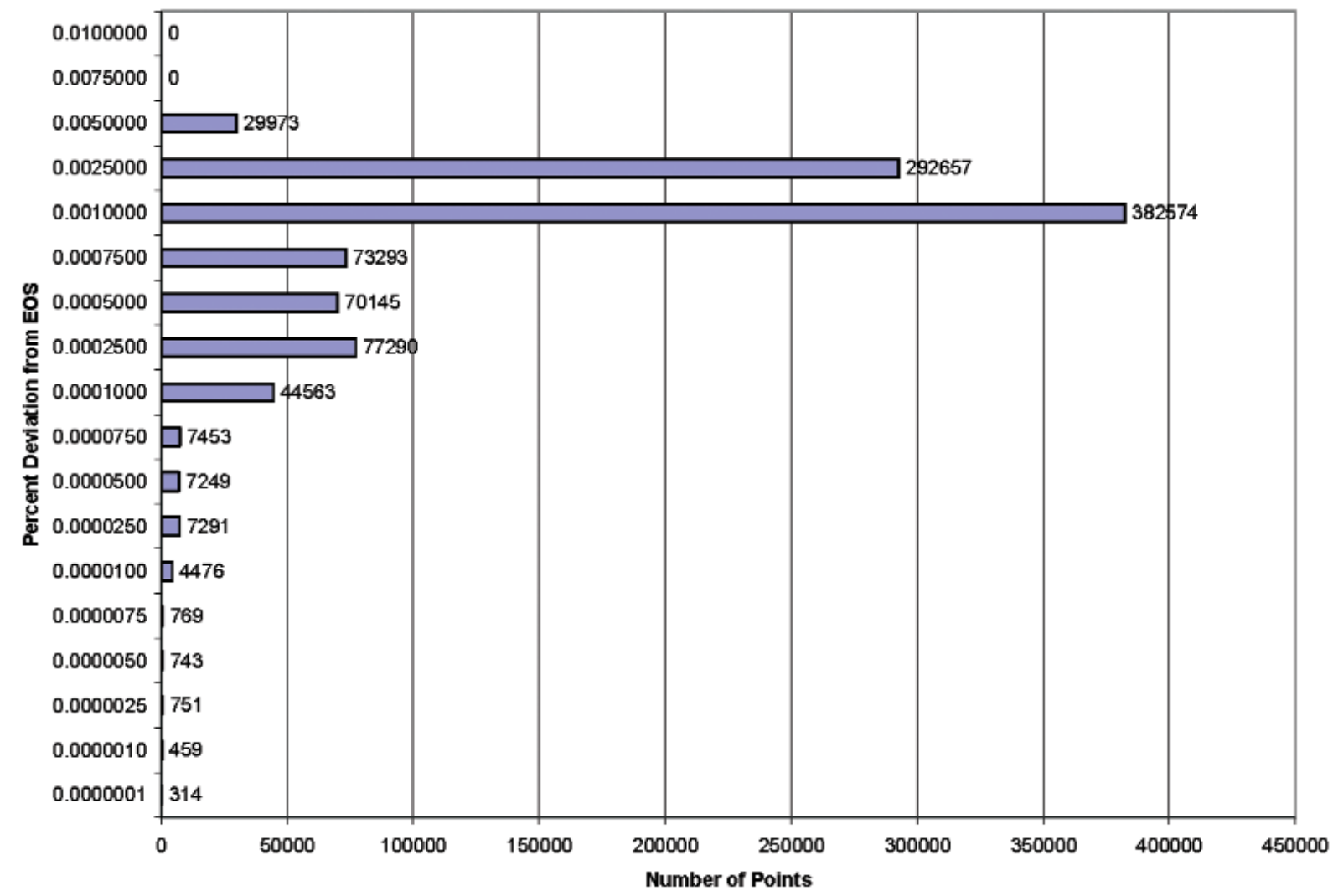

Fig. 1. Histogram illustrating the frequency of percentage density deviations for a sample containing one million points over the range of temperatures from $220 \mathrm{~K}$ to $1000 \mathrm{~K}$ with pressures to $70 \mathrm{MPa}$. 
Volume 113, Number 6, November-December 2008

Journal of Research of the National Institute of Standards and Technology

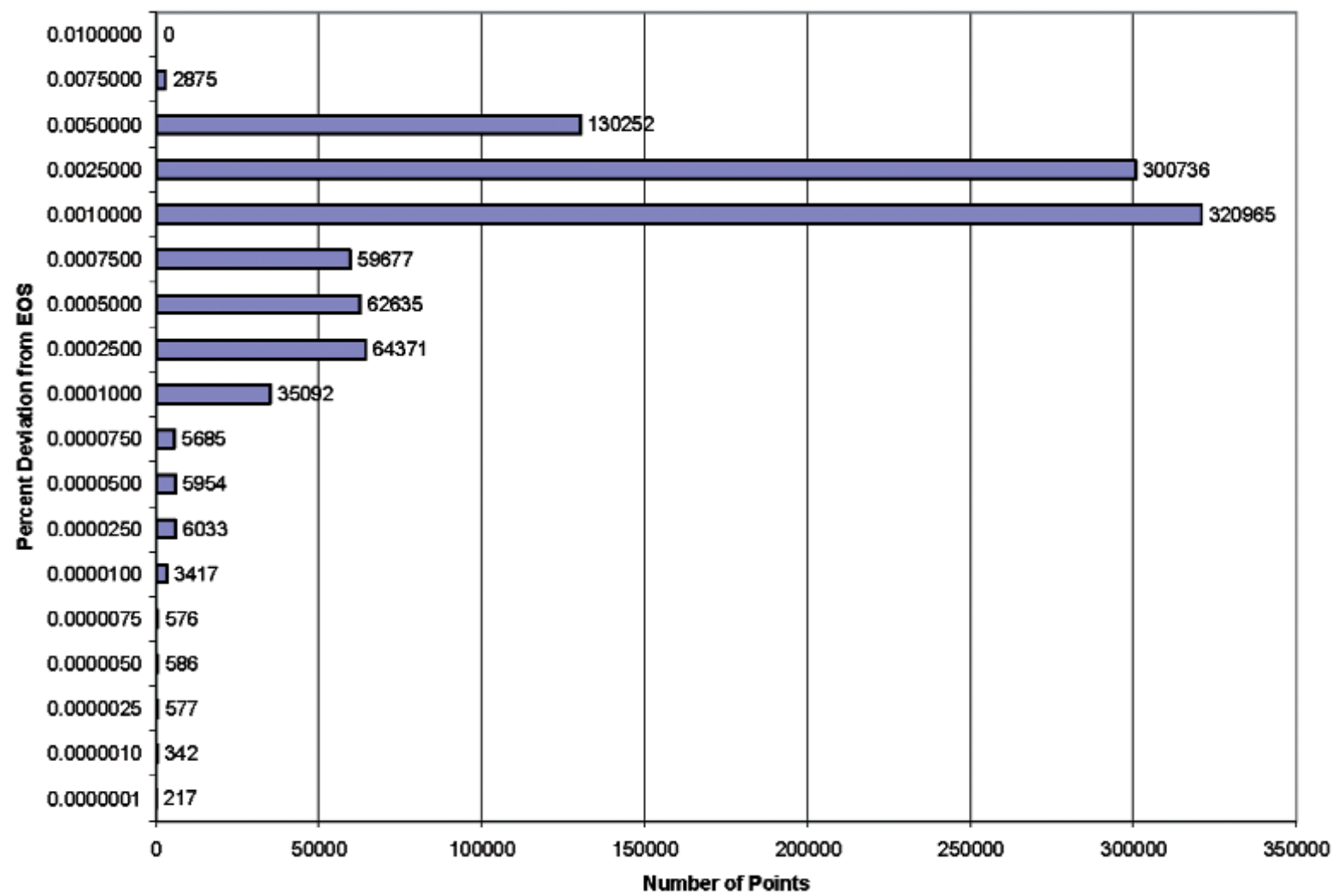

Fig. 2. Histogram illustrating the frequency of percentage density deviations for a sample containing one million points over the range of temperatures from $255 \mathrm{~K}$ to $1000 \mathrm{~K}$ with pressures to $120 \mathrm{MPa}$.

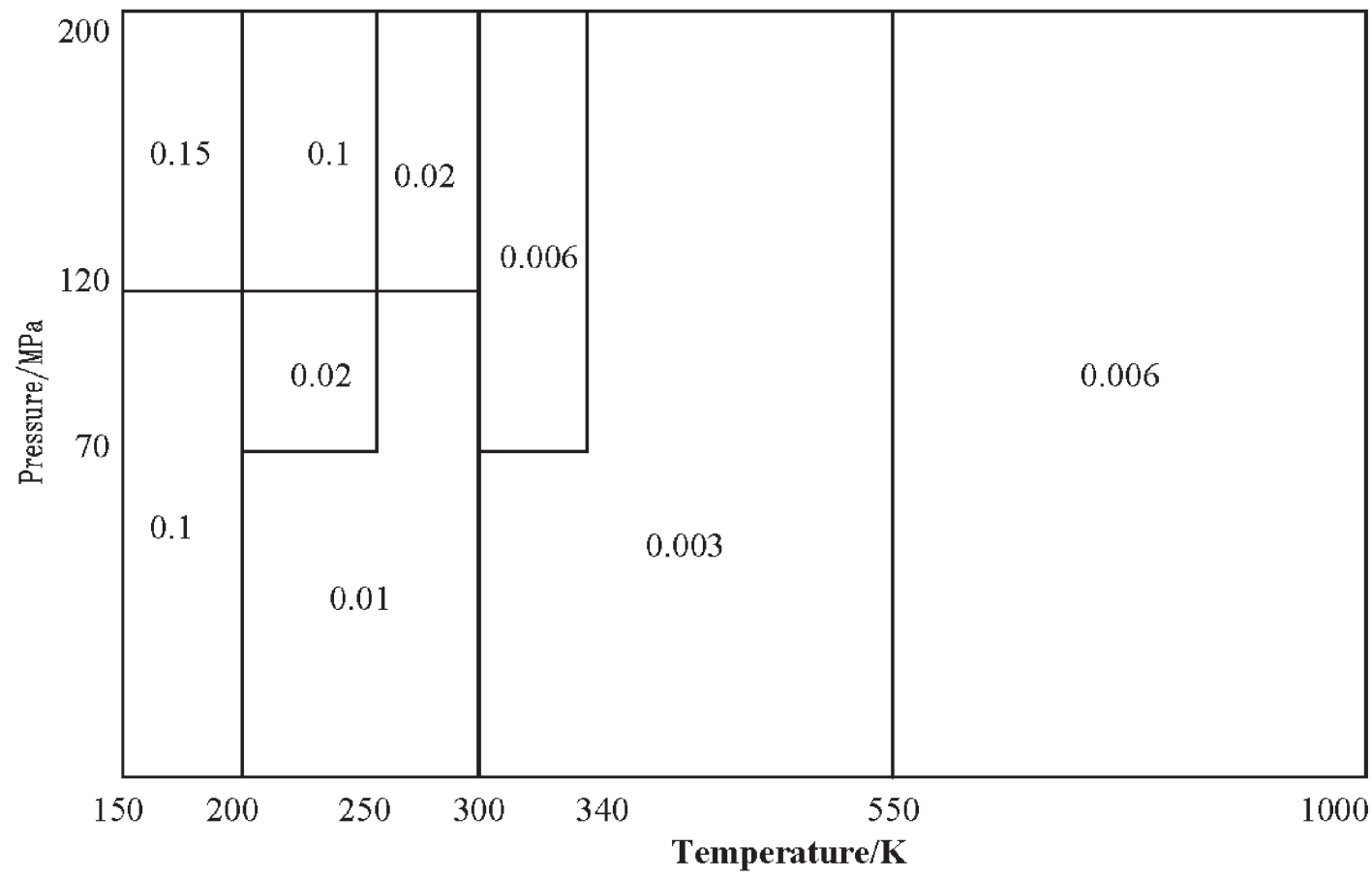

Fig. 3. Maximum percent differences between the equation of state of Leachman [4] and the equation presented here (axes are not to scale). 


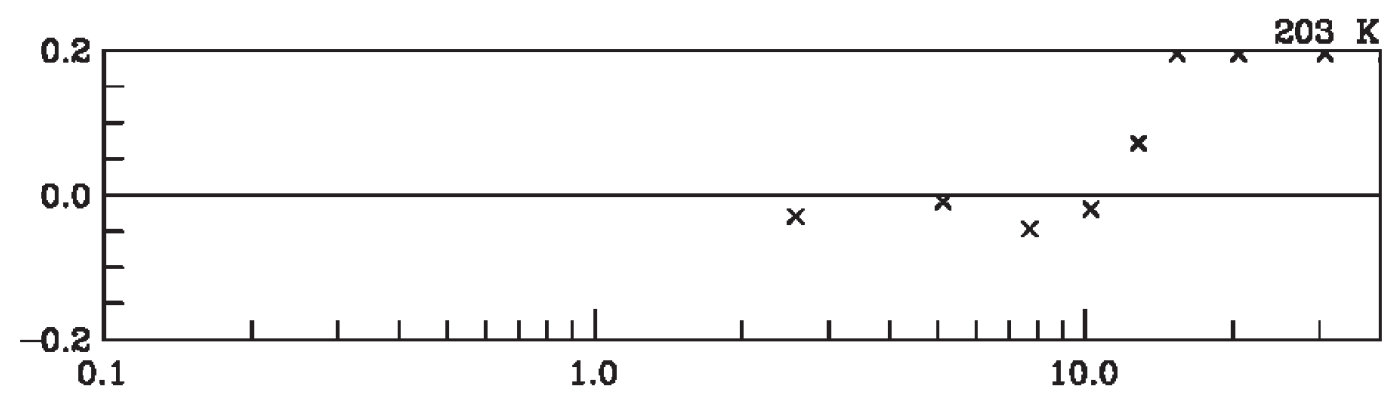

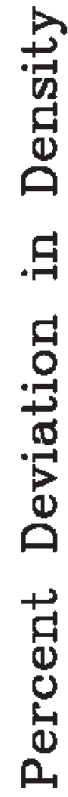
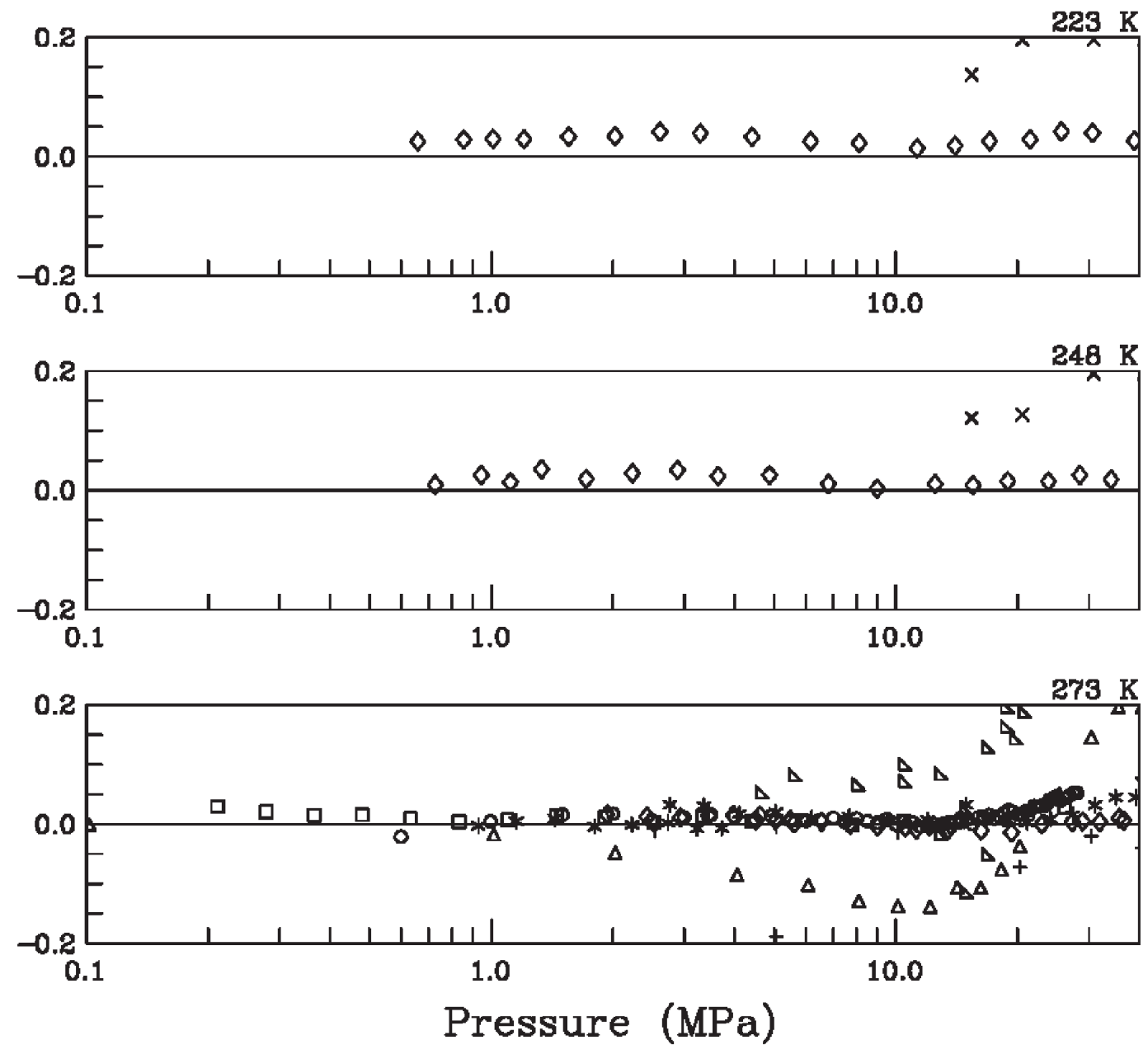

+ Bartlett et al. (1928)

$\times$ Bartlett et al. (1930)

a Jaeschke and Humphreys (1990) o GERG TM4 (1990) (Gasunie)

* Michels and Goudeket (1941)

$\Delta$ Townend and Bhatt (1931)

$\diamond$ Michels et al. (1959)

$Y$ Wiebe and Gaddy (1938)

Fig 4. Density deviations as a function of pressure for temperatures from $203 \mathrm{~K}$ to $273 \mathrm{~K}$. 

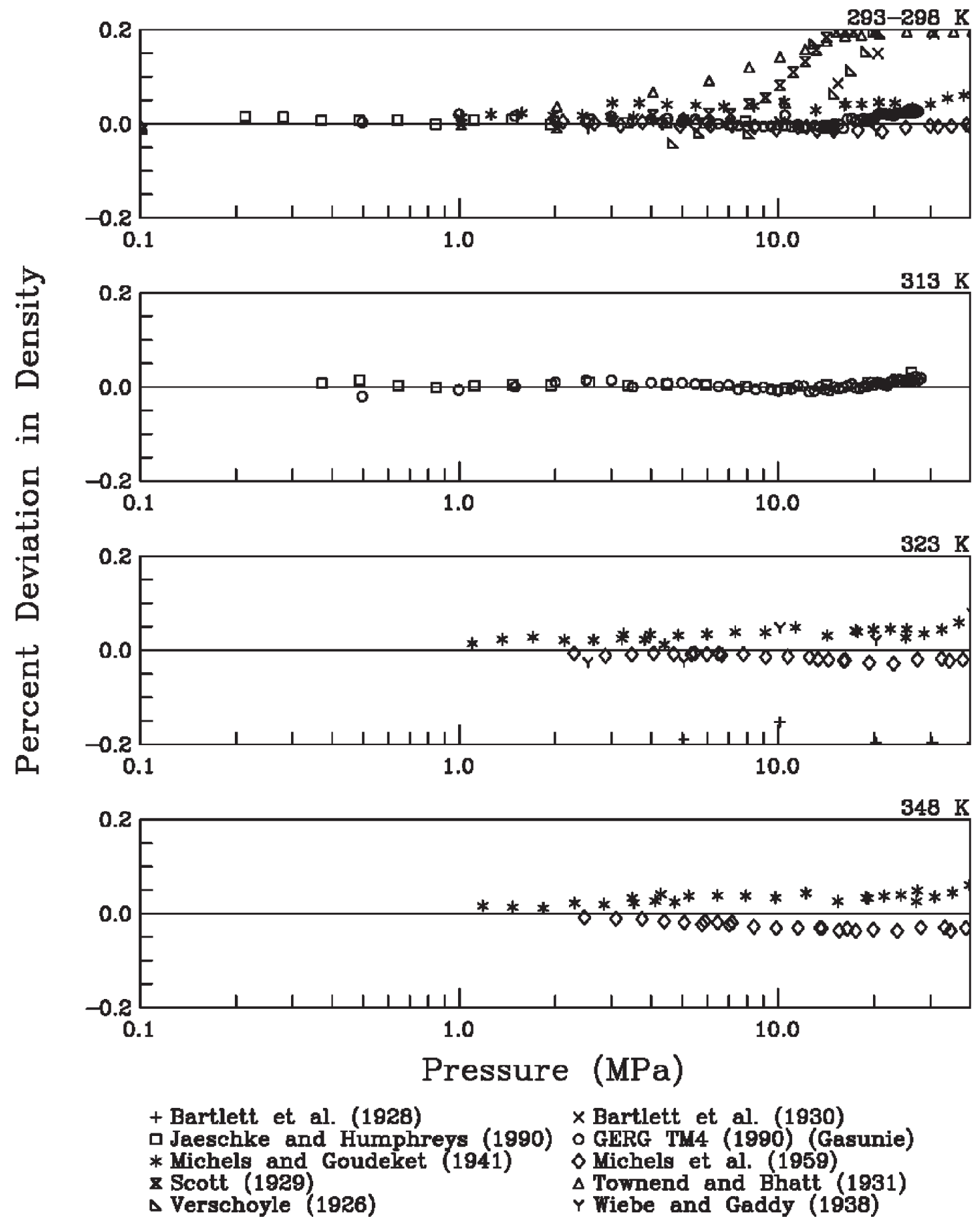

Fig. 5. Density deviations as a function of pressure for temperatures from $293 \mathrm{~K}$ to $348 \mathrm{~K}$. 

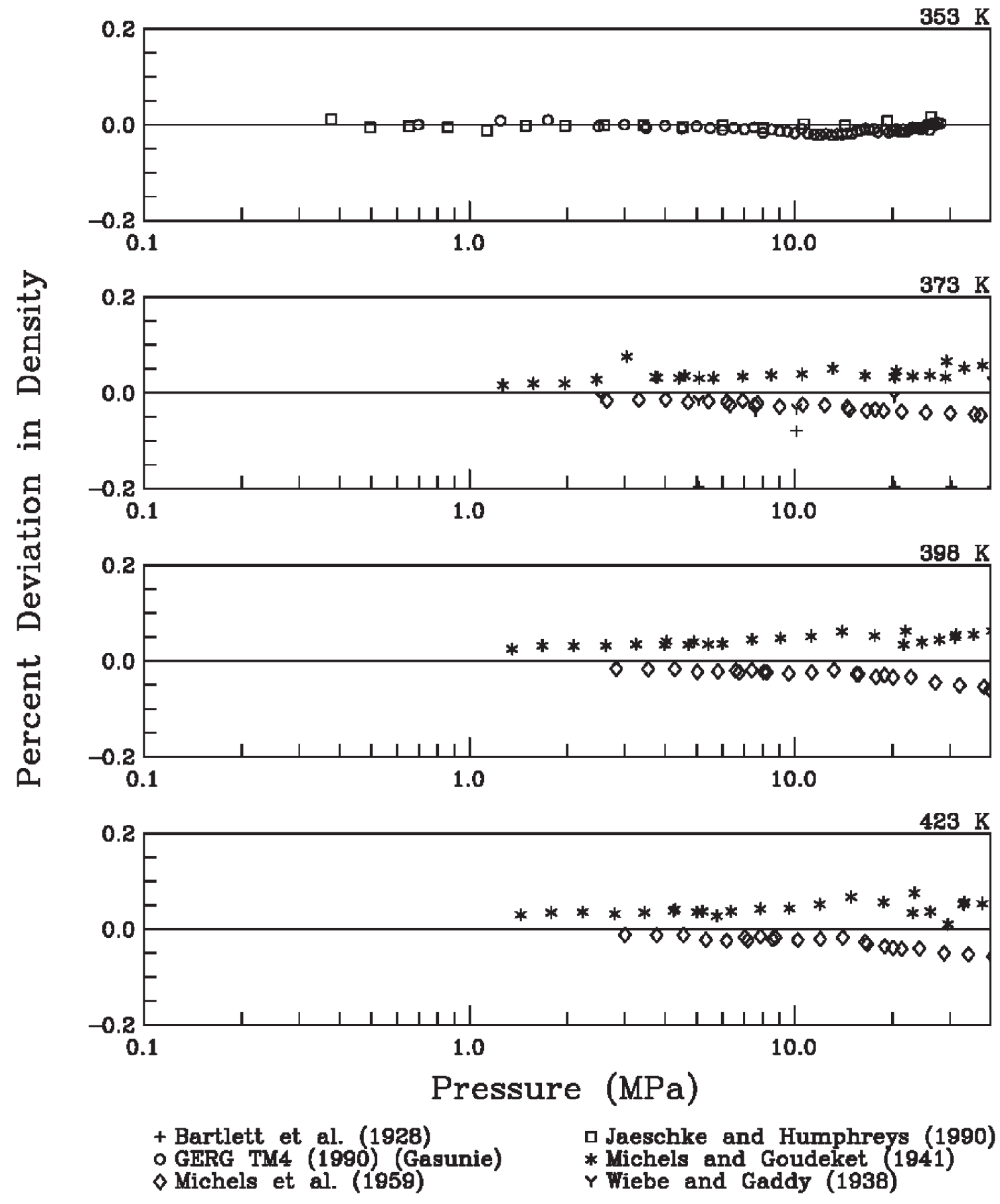

Fig. 6. Density deviations as a function of pressure for temperatures from $353 \mathrm{~K}$ to $423 \mathrm{~K}$. 
Several observations can be made from the deviation plots. These figures generally support the uncertainty estimate for the equation of state of Leachman [4]. The estimate of $0.04 \%$ as a combined uncertainty with coverage factor of two seems reasonable in this range considering only the scatter of the available density data and the agreement between the equation and these data. These observations support the use of Eq. (3) for fuel consumption calculations based on density calculations from initial and final pressures and temperatures.

\section{Conclusions}

Fuel consumption can be determined in multiple ways. From a regulatory view, it is mandatory to have consistent methods that can be used for independent verification. Equivalent measurement methods allow maximum flexibility in the regulated industry and confidence in data integrity. The determination of the mass of hydrogen gaseous fuel use is quite straightforward after the initial and final temperatures, pressures, and volumes have been accurately established. Consistent use of an expression for mass determination requires a standard method by which the compressibility factor can be calculated.

Equation (3) can form the basis of such a standard. It was developed to provide consistency with the calculations from a NIST Standard Reference Database [7], and it has been shown to provide reasonable agreement with the currently available data. The simple form of Eq. (3) agrees with the standard of Ref. [4] to within $0.01 \%$ from $220 \mathrm{~K}$ to $1000 \mathrm{~K}$ with pressures up to $70 \mathrm{MPa}$, and from $255 \mathrm{~K}$ to $1000 \mathrm{~K}$ with pressures up to $120 \mathrm{MPa}$, although the estimated uncertainty of the resulting hydrogen density is $0.04 \%$. The equation can be extended from $150 \mathrm{~K}$ to $1000 \mathrm{~K}$ with pressures up to $200 \mathrm{MPa}$ with an uncertainty of $0.15 \%$ at the lowest temperatures. The equation is not adequate for the calculation of other hydrogen properties. There might be other uncertainties in the quantification of hydrogen fuel consumption that have not been explicitly considered here. These could include the temperature and pressure dependence of the tank volume, equilibration of the temperature and pressure measurements, and other uncertainties in the measurement of temperature and pressure.

\section{Acknowledgments}

The authors thank Richard T Jacobsen and Steven G. Penoncello for their assistance given to Jacob
Leachman during the development of the equation of state for hydrogen. We also express our sincere thanks to Carl Paulina, USEPA-National Vehicle and Fuel Emissions Laboratory, for his enthusiasm and support of this project.

\section{References}

1. B. A. Younglove, J. Phys. Chem. Ref. Data, Suppl. 11, 1 (1982).

2. H. M. Roder and R. D. McCarty, A Modified Benedict-WebbRubin Equation of State for Parahydrogen-II, Natl. Bur. Stand., Interagency Rep. 75-814 (1975).

3. R. D. McCarty, J. Hord, and H. M. Roder, Selected Properties of Hydrogen (Engineering Design Data), Natl. Bur. Stand., Monogr. 168 (1981).

4. J. W. Leachman, Fundamental Equations of State for Parahydrogen, Normal Hydrogen, and Orthohydrogen, Master's Thesis, University of Idaho, Moscow (2007).

5. J. W. Leachman, R. T Jacobsen, E. W. Lemmon, and S. G. Penoncello, submitted to J. Phys. Chem. Ref. Data (2009).

6. E. W. Lemmon, M. L. Huber, D. G. Friend, and C. Paulina, Paper Number 2006-01-0434, SAE World Congress, Detroit MI, April 3-4 (2006).

7. E. W. Lemmon, M. L. Huber, and M. O. McLinden, NIST Standard Reference Database 23: Reference Fluid Thermodynamic and Transport Properties-REFPROP, Version 8.0, National Institute of Standards and Technology, Standard Reference Data Program, Gaithersburg (2007).

8. E. W. Lemmon and R. T Jacobsen, J. Phys. Chem. Ref. Data 34, 69 (2005)

9. M. E. Wieser, Pure Appl. Chem. 78, 2051 (2006).

10. P. J. Mohr, B. N. Taylor, and D. B. Newell, Rev. Mod. Phys. 80, 633 (2008)

11. E. P. Bartlett, H. L. Cupples, and T. H. Tremearne, J. Am. Chem. Soc. 50, 1275 (1928).

12. E. P. Bartlett, H. C. Hetherington, H. M. Kvalnes, and T. H. Tremearne, J. Am. Chem. Soc. 52, 1363 (1930).

13. M. Jaeschke and A. E. Humphreys, The GERG Databank of High Accuracy Compressibility Factor Measurements, GERG Technical Monograph 4, Verlag des Vereins Deutscher Ingenieure: Dusseldorf, Germany (1990).

14. A. Michels and M. Goudeket, Physica 8, 347 (1941).

15. A. Michels, W. De Graaff, T. Wassenaar, J. M. H. Levelt, and P. Louwerse, Physica 25, 25 (1959).

16. G. A. Scott, Proc. R. Soc. London A125, 330 (1929).

17. D. T .A. Townend and L. A. Bhatt, Proc. R. Soc. London A134, 502 (1931)

18. T. T .H. Verschoyle, Proc. R. Soc. A111, 552 (1926).

19. R. Wiebe and V. L. Gaddy, J. Am. Chem. Soc. 60, 2300 (1938).

About the authors: Eric W. Lemmon is a mechanical engineer and Marcia L. Huber is a chemical engineer in the Thermophysical Properties Division of the Chemical Science and Technology Laboratory at the National Institute of Standards and Technology. Jacob W. Leachman is a graduate student at the University of Wisconsin-Madison in the Cryogenics Lab. He received 
Volume 113, Number 6, November-December 2008

Journal of Research of the National Institute of Standards and Technology

his Master's degree at the Center for Applied Thermodynamic Studies (CATS) at the University of Idaho, Moscow, where the equation of state for hydrogen was in part produced. The National Institute of Standards and Technology is an agency of the U.S.

Department of Commerce. 